\title{
TIGHT BOUNDS ON THE ROUND COMPLEXITY OF DISTRIBUTED 1-SOLVABLE TASKS ${ }^{1}$
}

\author{
Ofer Biran \\ Science and Technology, IBM Israel \\ MATAM, Haifa, Israel 31905 \\ Shlomo Moran and Shmuel Zaks \\ Department of Computer Science \\ Technion, Haifa, Israel 32000
}

\begin{abstract}
A distributed task $T$ is 1-solvable if there exists a protocol that solves it in the presence of (at most) one crash failure. A precise characterization of the 1-solvable tasks was given in [BMZ]. In this paper we determine the number of rounds of communication that are required, in the worst case, by a protocol which 1-solves a given 1-solvable task $T$ for $n$ processors. We define the radius $R(T)$ of $T$, and show that if $R(T)$ is finite, then the number of rounds is $\Theta\left(\log _{n} R(T)\right)$; more precisely, we give a lower bound of $\log _{(n-1)} R(T)$, and an upper bound of $2+\left\lceil\log _{(n-1)} R(T)\right]$. The upper bound implies, for example, that each of the following tasks: renaming, order preserving renaming ([ABDKPR]) and binary monotone consensus ([BMZ]) can be solved in the presence of one fault in 3 rounds of communications. All previous protocols that 1 -solved these tasks required $\Omega(n)$ rounds. The result is also generalized to tasks whose radii are not bounded, e.g., the approximate consensus and its variants ([DLPSW , BMZ]).
\end{abstract}

\footnotetext{
${ }^{1}$ This research was supported in part by Technion V.P.R. Funds - Wellner Research Fund and Loewengart Research Fund, and by the Foundation for Research in Electronics, Computers and Communications, administrated by the Israel Academy of Sciences and Humanities, and by the ESPRIT II Basic Research Actions Program of the EC under contract no. 3075 (project ALCOM). A preliminary version of this paper appeared in the proceedings of the 4th International Workshop on Distributed Algorithms, Bari, Italy 1990.
} 


\section{INTRODUCTION}

An asynchronous distributed network consists of a set of processors, connected by communication lines, through which they communicate in order to accomplish a certain task; the time delay on the communication lines is finite, but unbounded and unpredictable. In this paper we study the case when at most one processor is faulty, which means that from some point on none of its messages is delivered (fail-stop failure). It was shown in [FLP] that it is impossible to achieve a distributed consensus for this case. This result was extended in several directions. In [DDS] the features of asynchrony that yield the result of [FLP] and related results were analyzed. The possibility of reaching agreement when restricting the pure asynchrony was studied also in [ADG,DLS]. In [DLPSW] it was shown that approximate consensus, in which all processors must agree on values that are arbitrarily close to one another, is possible in the presence of a constant fraction of faulty processors. In [ABDKPR] the solvability of two renaming problems (which will be defined later) in the presence of faults was investigated. In [MW] a class of tasks was shown not to be solvable in the presence of one faulty processor (not 1-solvable). In [BMZ] we provided a complete characterization of the 1-solvable tasks. Specifically, this characterization provides a halting procedure for deciding whether a given task, specified by its (finite) input/output relation, is 1-solvable. No such procedure is known for the case where $t>1$. A possible step towards this latter goal was done in three recent works [BG,HS,SZ], which relate the $t$-solvability of tasks (for arbitrary $t$ ) to properties of high dimensional simplicial complexes.

In this paper we are interested in the round complexity of a 1-solvable task, which is the number of communication rounds that are required, in the worst case, by any protocol that 1solves it. This measure attempts to capture the notion of time complexity for asynchronous, fault tolerant protocols. In [Fe], a tight bound was given for the specific task of the approximate consensus. Results of the same type in other models were given in [ALS] for the approximate consensus task in asynchronous shared memory model, and in [HT] for the renaming task in synchronous message passing model.

We provide optimal bounds (up to an additive constant) on the round complexity of a general 1-solvable task. We first consider bounded tasks, which are tasks that can be 1-solved by protocols that require at most a constant number of rounds in all possible executions (e.g., the renaming tasks and the strong binary monotone consensus task [ABDKPR, BMZ]). Then we generalize our results for unbounded tasks (like the approximate consensus and its variants [DLPSW , BMZ]).

The outline of our proof is as follows: For a distributed task $T$, let $X_{T}$ be the set of possible input vectors for $T$. First we show, by using the result in [BMZ], that if $T$ is 1-solvable, then there is a set $\boldsymbol{R}_{T}$ of radius functions related to $T$, where each radius function $\rho$ is a mapping $\rho: X_{T} \rightarrow N$, which maps each input vector $\vec{x}$ to a positive integer $\rho(\vec{x})$. We use this set to define $R(T)$, the radius of the task $T$, as $R(T)=\min _{\rho \in \boldsymbol{R}_{T}} \sup _{\vec{x} \in X_{T}} \rho(\vec{x})$.

In proving our bounds, we first consider only tasks $T$ for which $R(T)$ is finite, and show that these are exactly the bounded tasks. We show that if $R(T)$ is finite then the round complexity of $T$ is $\Theta\left(\log _{n} R(T)\right)$; more precisely, we give a lower bound of $\log _{(n-1)} R(T)$, and an 
upper bound of $2+\left\lceil\log _{(n-1)} R(T)\right]$. We then extend the results to an arbitrary task $T$. In the general case, the round complexity of $T$ is not a constant, but a function of the input vector. Since there is no natural total order on these functions, we cannot define the optimal round complexity of $T$, but only define the set of minimal round complexity functions of $T$, in the natural partial ordering of functions. This set is defined by a correspondence to the set of minimal radius functions in $\boldsymbol{R}_{T}$.

The upper bound implies, for example, that each of the following tasks: renaming with $n+1$ new names, order preserving renaming with $2 n-1$ new names ([ABDKPR]), and strong binary monotone consensus ([BMZ]) can be solved in the presence of one fault in three rounds of communications. All previous protocols that 1-solved these tasks required $\Omega(n)$ rounds (however, it is fair to note that the protocols for the renaming problems [ABDKPR] were designed to tolerate multiple failures). For the case where $R(T)$ is infinite, we extend the optimal bounds of [Fe] for the approximate consensus: we show that similar bounds hold for variants of the approximate consensus that were studied in [BMZ], which are considerably harder than the (original) approximate consensus.

The rest of the paper is organized as follows: In Section 2 we provide the preliminary definitions. In Section 3 we define standard protocols and round complexity. In Section 4 we define the radius of a task. The lower and upper bounds for bounded tasks are presented in Sections 5 and 6. In Section 7 we generalize our results for arbitrary tasks and in Section 8 we present some applications.

\section{PRELIMINARY DEFINITIONS AND NOTATIONS}

\subsection{Asynchronous Systems}

An asynchronous distributed system is composed of a set $P=\left\{P_{1}, P_{2}, \ldots, P_{n}\right\}$ of $n$ processors $(n \geq 3)$, each having a unique identity. We assume that the identities of the processors are mutually known, and w.l.o.g. that the identity of $P_{i}$ is $i$. Our results are applicable also to the model in which the identities are not mutually known; the modification of the results to this model is done in a way similar to the one described in [BMZ]. The processors are connected by communication links, and they communicate by exchanging messages along them. Messages arrive with no error in a finite but unbounded and unpredictable time; however, one of the processors might be faulty, in which case messages might not have these properties (the exact definition is given in the sequel).

\subsection{Decision Tasks}

Definition: Let $X$ and $D$ be sets of input values and decision values, respectively. A distributed decision task $T$ is a function

$$
T: X_{T} \rightarrow 2^{D^{n}}-\{\varnothing\}
$$

where $X_{T} \subseteq X^{n} . X_{T}$ is called the input set of the task T. $D_{T}$, the decision set of the task $T$, is the union of the sets $T(\vec{x})$ over all $\vec{x} \in X_{T}$. Each vector $\vec{x}=\left(x_{1}, x_{2}, \ldots, x_{n}\right) \in X_{T}$ is called an input vector, and it represents the initial assignment of the input value $x_{i} \in X$ to processor $P_{i}$, for $i=1,2, \ldots, n$. Each vector $\vec{d}=\left(d_{1}, d_{2}, \ldots, d_{n}\right) \in D_{T}$ is called a decision vector, and it represents 
the assignment of a decision value $d_{i} \in D$ to processor $P_{i}$, for $i=1,2, \ldots, n$.

Thus, a decision task $T$ maps each input vector to a non-empty set of allowable decision vectors. We assume that all tasks $T$ discussed in this paper are computable, in the sense that the set $\left\{(\vec{x}, \vec{d}): \vec{x} \in X_{T}\right.$ and $\left.\vec{d} \in T(\vec{x})\right\}$ is recursive.

\section{Examples:}

(1) Consensus [FLP]: A consensus task is any task $T$ where $X_{T}=X^{n}$ for an arbitrary set $X$, and such that $T(\vec{x}) \subseteq\{(0,0, \ldots 0),(1,1, \ldots, 1)\}$ for every input vector $\vec{x} \in X_{T}$. Let $\overrightarrow{0}$ denote the vector $(0,0, \ldots, 0)$, and $\overrightarrow{1}$ denote the vector $(1,1, \ldots, 1)$. A strong consensus task is a consensus task $T$, in which there exist two input vectors $\vec{u}$ and $\vec{v}$ such that $T(\vec{u})=\{\overrightarrow{0}\}$ and $T(\vec{v})=\{\overrightarrow{1}\}$. The main result in [FLP] implies that a strong consensus task is not 1-solvable.

(2) Strong Binary Monotone Consensus [BMZ]: This is probably the strongest variant of the consensus task which is 1-solvable. To simplify the definition, assume that $n$ is even: The input is an integer vector $\vec{x}=\left(x_{1}, \cdots, x_{n}\right)$, and $T(\vec{x})$ consists of all vectors $\vec{d}=\left(d_{1}, \cdots, d_{n}\right)$ where each $d_{i}$ is one of the two medians of the multiset $\left\{x_{1}, \cdots, x_{n}\right\}$, and $d_{i} \leq d_{i+1}$ (the "strong" stands for the fact that the two values must be the medians).

(3) Renaming [ABDKPR]: This task is defined for a given integer $K$, where $K \geq n$. The input set $X_{T}$ is the set of all vectors $\left(x_{1}, \cdots, x_{n}\right)$ of distinct integers. For a given input $\vec{x}, T(\vec{x})$ is the set of all integer vectors $\vec{d}=\left(d_{1}, \cdots, d_{n}\right)$ satisfying $1 \leq d_{i} \leq K$ and such that for each $i, j, d_{i} \neq d_{j}$. In order to prevent trivial solutions in which $P_{i}$ always decides on $i$, this task assumes a model in which the processors identities are not known.

(4) Order Preserving Renaming (OPR) [ABDKPR]: This task is similar to the renaming task, with the additional requirement that for each $i, j, x_{i}<x_{j}$ implies $d_{i}<d_{j}$.

(5) Approximate Consensus [DLPSW]: This task is defined for any given $\varepsilon>0$. The input set $X_{T}$ is $Q^{n}$, where $Q$ is the set of rational numbers, and for a given input $\vec{x}=\left(x_{1}, \cdots, x_{n}\right), T(\vec{x})$ is the set of all vectors $\vec{d}=\left(d_{1}, \cdots, d_{n}\right)$ satisfying $\left|d_{i}-d_{j}\right| \leq \varepsilon$ and $m \leq d_{i} \leq M \quad(1 \leq i, j \leq n)$, where $m=\min \left\{x_{1}, \cdots, x_{n}\right\}$ and $M=\max \left\{x_{1}, \cdots, x_{n}\right\}$.

(6) Strong Binary Monotone Approximate Consensus [BMZ]: This is a harder variant of the approximate consensus task which is still 1-solvable. To simplify the definition, assume that $n$ is even: The input is the same as for the approximate consensus. For an input $\vec{x}=\left(x_{1}, \cdots, x_{n}\right), T(\vec{x})$ consists of all vectors $\vec{d}=\left(d_{1}, \cdots, d_{n}\right)$ satisfying: $\vec{d}$ has at most two distinct entries, which lie between the two medians of the multiset $\left\{x_{1}, \cdots, x_{n}\right\}$, and $d_{i} \leq d_{i+1} \leq d_{i}+\varepsilon$.

\subsection{Protocols and Executions}

A protocol for a given network is a set of $n$ programs, each associated with a single processor in the network. Each such program contains operations of sending a message to a neighbor, receiving a message and processing information in the local memory. The local processing includes a special operation called deciding, which the processor may execute only once; A processor decides by writing a decision value to a write-once register.

If the network is initialized with the input vector $\vec{x} \in X^{n}$ (i.e., the value $x_{i}$ is assigned to processor $P_{i}$ ), and if each processor executes its own program in a given protocol $\alpha$, then the sequence of operations performed by the processors is called an execution of $\alpha$ on input $\vec{x}$. (We assume here that no two operations occur simultaneously; otherwise, we order them arbitrarily. For more formal definitions see, e.g., [KMZ]. For the definition of the atomic step we adapt the model of [FLP].)

Definition: A vector $\vec{d}=\left(d_{1}, d_{2}, \cdots, d_{n}\right)$ is an output vector of $\alpha$ on input $\vec{x}$ if there is an execution of $\alpha$ on $\vec{x}$ in which processor $P_{i}$ decides on $d_{i}$, for $i=1 \cdots n$. 


\subsection{Faults and 1-Solvability}

Definition: A processor $P$ is faulty in an execution $e$ if it stops participating in the protocol prematurely (a fail-stop failure; see, e.g., [FLP]. Also known as crash failure; see, e.g., [NT]). A processor is non-faulty otherwise.

Definition: A protocol $\alpha$ 1-solves a task $T$ if for every execution of $\alpha$ on any input $\vec{x} \in X_{T}$ in which at most one processor is faulty, the following two conditions hold:

(1) All the non-faulty processors eventually decide.

(2) If no processor is faulty in the execution, then the output vector belongs to $T(\vec{x})$.

When such a protocol $\alpha$ exists we say that the task $T$ is $1-$ solvable.

The definition above does not require the processors to halt after reaching a decision. However, in the case of a single failure, it is not hard to see that a processor that learns that $n-1$ processors (including itself) have already decided may halt. Hence, in this case, reaching a decision by all non-faulty processors is sufficient to guarantee halting. For this reason, in this paper we shall restrict the discussion to protocols in which the processors are guaranteed to halt in every possible execution. (Note that in the case of $t>1$ crash failures, there exist tasks which can be $t$-solved only by protocols that do not guarantee termination, e.g, the renaming tasks [ABDKPR]. For more on the termination requirement for multiple failures see [TKM]).

\section{STANDARD PROTOCOLS AND ROUND COMPLEXITY}

In this paper we bound the number of communication rounds that are required by protocols that 1-solve a given task. This number attempts to capture the notion of time complexity for asynchronous fault tolerant protocols. We model an arbitrary $t$-resilient protocol that works in rounds of communications by the notion of standard protocol. The definitions and discussion below are restricted to the case $t=1$.

\subsection{Standard Protocols}

A protocol that 1-solves a task $T$ is a standard protocol if it works in rounds of communications, as follows. In each round a processor broadcasts a message (which includes the round number), which is a function of its state, to all the processors (including itself), and waits until it receives $n-1$ messages of this round (including its own message which is received first; it may wait for less than $n-1$ messages if it heard of processors that had already halted). During this period of waiting, it might receive messages from different rounds. Those of higher rounds are saved until the processor itself reaches these rounds. Messages of previous rounds (there can be at most one such message from each previous round) are gathered with the $n-1$ messages of this round to form a set $M$. Then the processor computes its next state, which is a function of $M$ and its previous state. The state of a processor includes its write-once register. Our notion of standard protocol is similar to the one used in $[\mathrm{Fe}]$.

Formally, the standard protocol for $P_{k}$ is as follows:

$r \leftarrow 0$

state $\leftarrow$ INIT_k

while state $\neq$ HALT do 
$r \leftarrow r+1$

$\operatorname{BROADCAST}\left(r, M E S S A G E \_F U N C T I O N \_k(\right.$ state $\left.)\right)$

WAIT until you RECEIVE ( $n-1-$ [\# of known halted processors]) messages of the form $(r, *)$

$M \leftarrow\left\{m \mid\right.$ a message $\left(r^{\prime}, m\right), r^{\prime} \leq r$ was received in the above WAIT, or a message $(r, m)$ was received in a previous round $\}$

state $\leftarrow S T A T E \_F U N C T I O N \_k($ state,$M)$

end

\subsection{Round Complexity}

Definition: Let $T$ be a task and $\alpha$ a standard protocol that 1 -solves $T$. The round complexity of $\alpha$ on input $\vec{x}$, denoted $r c_{\alpha}(\vec{x})$, is the maximum round number, over all executions of $\alpha$ on input $\vec{x}$, that a non-faulty processor reaches.

The round complexity of $\alpha$, denoted $r c_{\alpha}(T)$ is defined by: $r c_{\alpha}(T)=\sup _{\vec{x} \in X_{T}} r c_{\alpha}(\vec{x})$.

The round complexity $r c(T)$ of a task $T$ is defined by: $r c(T)=\min \left\{r c_{\alpha}(T) \mid \alpha 1\right.$-solves $\left.T\right\}$.

Note that $r c(T)$ may be infinite; this is the case only when the input set $X_{T}$ is infinite, and for any protocol $\alpha$ that 1 -solves $T$ and for any constant $C$, there is an input $\vec{x}$ such that $r c_{\alpha}(\vec{x})>C$.

Definition: A 1-solvable task $T$ is bounded if $r c(T)$ is finite, and is unbounded otherwise.

We will first present results for bounded tasks, and then extend them to results which are applicable for unbounded tasks as well.

\section{COVERING FUNCTIONS AND RADII OF TASKS}

We first give some basic definitions from [BMZ] which are needed for this paper.

\subsection{Adjacency Graphs, Partial Vectors, Covering Vectors and i-Anchors}

Definition: Let $S \subseteq A^{n}$, for a given set $A$. Two vectors $\vec{s}_{1}, \vec{s}_{2} \in S$ are adjacent if they differ in exactly one entry. The adjacency graph of $S, G(S)=\left(S, E_{S}\right)$, is an undirected graph, where $\left.\vec{s}_{1}, \vec{s}_{2}\right) \in E_{s}$ iff $\vec{s}_{1}$ and $\vec{s}_{2}$ are adjacent. For a task $T$ and an input vector $\vec{x}$ for $T, G(T(\vec{x}))$ is the decision graph of $\vec{x}$.

Definition: A partial vector is a vector in which one of the entries is not specified; this entry is denoted by ' $*$ '. For a vector $\vec{s}=\left(s_{1}, \cdots, s_{n}\right), \vec{s}^{i}$ denotes the partial vector obtained by assigning $*$ to the $i$-th entry of $\vec{s}$, i.e., $\vec{s}^{i}=\left(s_{1}, \cdots, s_{i-1}, *, s_{i+1}, \cdots, s_{n}\right) . \vec{s}$ is called an extension of $\vec{S}^{i}$.

Definition: Let $\vec{x}^{i}$ be a partial input vector and $\vec{d}^{i}$ a partial decision vector of a task $T$. We say that $\vec{d}^{i}$ is a covering vector for $\vec{x}^{i}$ if for each extension of $\vec{x}^{i}$ to an input vector $\vec{x} \in X_{T}$, there is an extension of $\vec{d}^{i}$ to a decision vector $\vec{d} \in T(\vec{x})$.

Note that in an execution on input $\vec{x}$ in which the messages of $P_{i}$ are delayed, the remaining $n-1$ processors must eventually output a covering vector for $\vec{x}^{i}$. If eventually $P_{i}$ decides 
too, then the resulting output vector is an $i$-anchor, which we define below.

Definition: A vector $\vec{d}$ is an $i$-anchor of an input vector $\vec{x}$ if $\vec{d} \in T(\vec{x})$ and $\vec{d}^{i}$ is a covering vector for $\vec{x}^{i}$.

Example: Consider the $O P R$ task (defined in Section 2.2) for $n=3$ processors and $K=5$. For the partial input vector $\vec{x}^{2}=(10, *, 30)$ there is a unique covering vector $\vec{d}^{2}=(2, *, 4)$, and the input vector $\vec{x}=(10,20,30)$ has a unique 2 -anchor $\vec{d}=(2,3,4)$. In the $O P R$ task with $n=3$ and $K=6$ there are three covering vectors for $\vec{x}^{2}:(2, *, 4),(2, *, 5)$, and $\left(3,{ }^{*}, 5\right)$. Thus, $\vec{x}$ has four 2 anchors: $(2,3,4),(2,3,5),(2,4,5)$ and $(3,4,5)$.

\subsection{Covering Functions and Radii of Tasks}

Definition: A covering function for a given task $T$ is a function that maps each partial input vector to a corresponding covering vector for it.

Definition: Let $T$ be a task, $C F$ a covering function for $T$, and $\vec{x} \in X_{T}$ an input vector. An anchors tree for $\vec{x}$ based on $C F$ is a tree in $G(T(\vec{x}))$ that, for each $i(1 \leq i \leq n)$, includes an $i$ anchor which is an extension of $C F\left(\vec{x}^{i}\right)$.

We now reformulate Theorem 3 of [BMZ] to a form suitable to our discussion:

Theorem [BMZ] : A task $T$ is 1-solvable if and only if there exists a covering function $C F$ for $T$, such that for each input vector $\vec{x} \in X_{T}$, there is an anchors tree for $\vec{x}$ based on $C F$.

A covering function satisfying the condition of Theorem [BMZ] is termed a solving covering function for $T$. As we show in Section 6, such functions may be used to construct protocols that 1-solve $T$.

Each solving covering function $C F$ defines a radius function $\rho_{C F}: X_{T} \rightarrow N$, as follows.

Definition: Let $C F$ be a solving covering function for $T$, and $\vec{x}$ an input vector in $X_{T} \cdot \rho_{C F}(\vec{x})$ is the minimum possible radius of an anchors tree for $\vec{x}$ based on $C F .^{2}$

The set of all radius functions for $T$ is denoted by $\boldsymbol{R}_{T}$. That is,

$$
\boldsymbol{R}_{T}=\left\{\rho_{C F}: C F \text { is a solving covering function for } T\right\} .
$$

Definition: $R(T)$, the radius of the task $T$, is given by:

$$
R(T)=\min _{\rho_{C F} \in \boldsymbol{R}_{T}} \sup _{\vec{x} \in X_{T}} \rho_{C F}(\vec{x}) .
$$

A covering function $C F$, and the corresponding radius function $\rho_{C F}$, are optimal for a task $T$ if $R(T)$ is finite, and $\max _{\vec{x} \in X_{T}} \rho_{C F}(\vec{x})=R(T)$.

Note that $R(T)$ may be infinite. This is the case only when the input set $X_{T}$ is infinite, and for any radius function $\rho_{C F}$ in $\boldsymbol{R}_{T}$ and for any constant $C$, there is an input $\vec{x}$ such that $\rho_{C F}(\vec{x})>C$. As we shall show, $R(T)$ is finite iff $T$ is a bounded task.

Example: Consider the following task $T$ for $n=3$ processors, in which $X_{T}$ contains only 3 input

${ }^{2}$ The radius of a tree $T$ is the minimal integer $r$, such that for some vertex $v$ in $T$, every vertex in $T$ is at distance at most $r$ from $v$. 
vectors:

$\vec{x}_{1}=(50,20,30), \vec{x}_{2}=(10,20,30)$ and $\vec{x}_{3}=(10,20,70)$.

$T\left(\vec{x}_{1}\right)=\{(5,2,3)\}$,

$T\left(\vec{x}_{2}\right)=\{(1,2,3),(1,4,3),(5,4,3),(5,4,6),(7,4,6),(7,5,6),(7,5,8),(3,5,8),(3,2,8)\}$ and

$T\left(\vec{x}_{3}\right)=\{(7,4,1),(3,2,1)\}$ (see Figure 1$)$

Now, in choosing an optimal covering function for $T$, the only partial input vectors that should be considered are those which might be extended to more than one input vector (if $\vec{x}^{i}$ might be extended to a unique input vector $\vec{x}$, then any vector $\vec{d}$ in $T(\vec{x})$ is an $i$-anchor of $\vec{x}$, so the need to select an $i$-anchor does not impose any constraint on the anchors tree). Thus we consider only $(*, 20,30)$ and $(10,20, *)$, so the only anchors that constrain the anchors tree are the 1 -anchor and the 3 -anchor. 


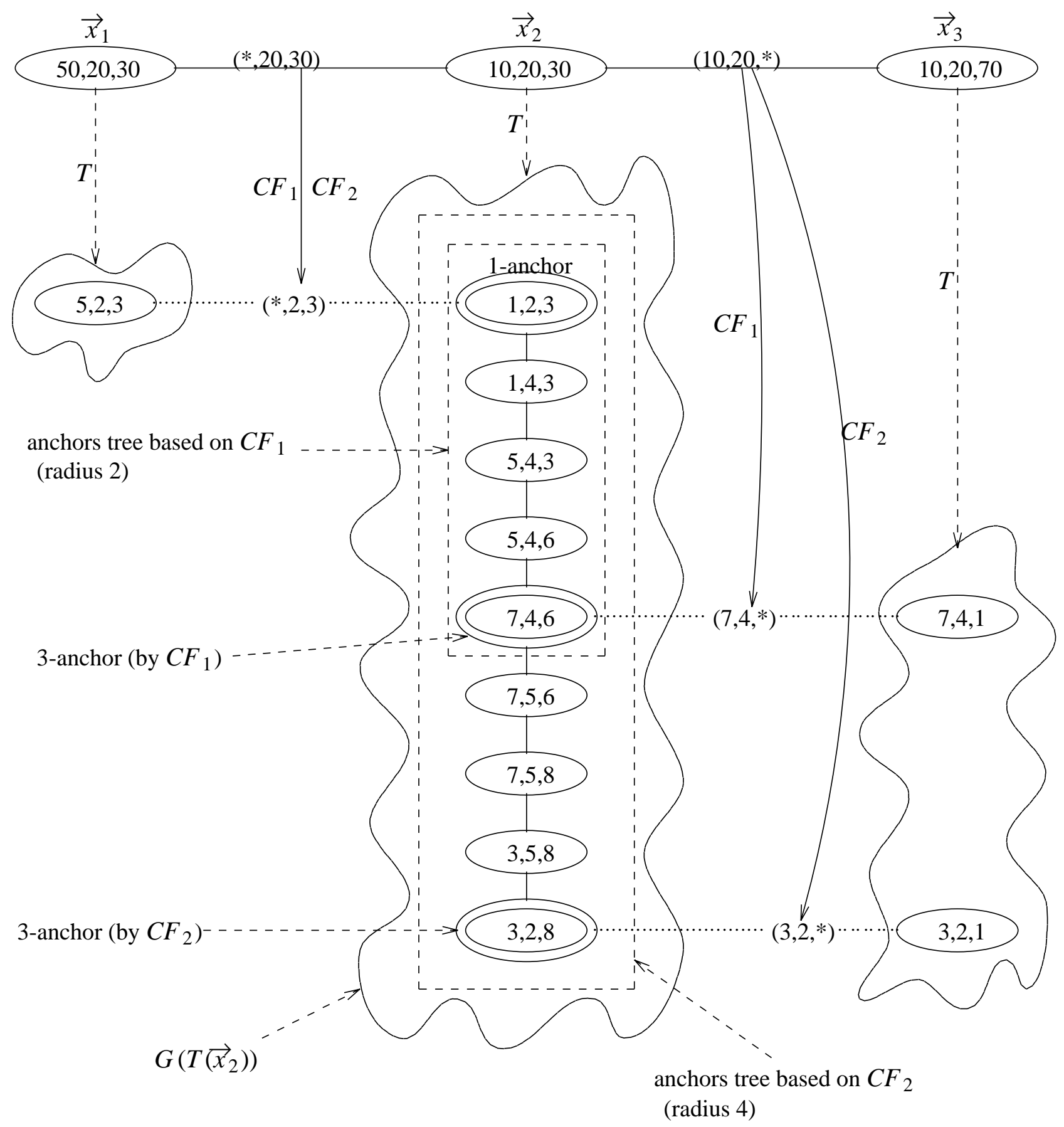

Figure 1: A task $T$ with $R(T)=2\left(=\rho_{C F_{1}}\left(\vec{x}_{2}\right)\right)$

From the decision graphs in Figure 1, clearly $R(T)$ is determined by $T\left(\vec{x}_{2}\right)$, since any anchors tree of the other two input vectors is composed of a single vertex. Based on the previous discussion, it suffices to consider only two covering functions, $C F_{1}$ and $C F_{2}$, whose values on the two "key" partial vectors are as follows:

$C F_{1}((*, 20,30))=(*, 2,3), C F_{1}\left(\left(10,20,{ }^{*}\right)\right)=(7,4, *)$ and $C F_{2}((*, 20,30))=(*, 2,3), C F_{2}((10,20, *))=(3,2, *)$.

In the minimum radius anchors tree based on $C F_{1}$ (in $\left.G\left(T\left(\vec{x}_{2}\right)\right)\right)$ the 1-anchor is $(1,2,3)$, the 
3 -anchor is $(7,4,6)$, and thus the radius is 2 (a line, with center $(5,4,3)$ ). The anchors tree based on $C F_{2}$ has the same 1 -anchor, its 3 -anchor is $(3,2,8)$, and its radius is 4 . So $C F_{1}$ is the optimal covering function, and $R(T)=2$.

More examples appear in Section 8.

\section{LOWER BOUND}

In this section we prove the following theorem.

Theorem 1: Let $T$ be a bounded task. Then its round complexity $r c(T)$ satisfies

$$
r c(T) \geq \log _{(n-1)} R(T) .
$$

Proof: Let $\alpha$ be a standard protocol which 1-solves $T$, and $r c_{\alpha}(T)=s$. We will prove that $\alpha$ implies a solving covering function for $T, C F_{\alpha}$, such that $\rho_{C F_{\alpha}}(\vec{x}) \leq(n-1)^{s}$ for every input vector $\vec{x}$, and thus $R(T) \leq(n-1)^{s}$. To simplify the presentation, we assume that in all executions of $\alpha$ no processor halts before round $s$ (and hence that all processors halt in round $s$ ). Clearly, such an assumption does not affect the generality of the proof, since we can always modify $\alpha$ such that processors that halt in round $r<s$ will continue to send "dummy" messages in later rounds. Note that this assumption enables us to assume that in each round, each processor waits for exactly $n-1$ messages (including its own message) of this round.

For the proof we construct sequences of executions of $\alpha$, which first require some definitions and discussion.

Definition: $e$ is an $r$-round execution of a standard protocol $A$ if $e$ is the first $r$ rounds of an execution of $A . e$ is an $r$-round $i$-sleeping execution if during $e$, no processor $P_{j}, j \neq i$, ever receives a message from $P_{i}$.

Let $e$ be an $r$-round execution of $\alpha$, and let $1 \leq l \leq r$. The $l$-senders of $P_{k}$ in $e$ is the set of processors from which $P_{k}$ receives messages $(l, *)$ in the $l$ 'th round of $e$. Note that the $l$ senders of $P_{k}$ always contains $P_{k}$, and that its cardinality is $n-1$.

Definition: An $r$-round execution $e$ is an ordered execution if for each $1 \leq k \leq n$ and for each $1 \leq l \leq r$, each processor $P_{k}$ receives in round $l$ exactly all the messages $(t, *), t \leq l$, sent to it by its $l$-senders, and which it has not received yet.

All the executions discussed in the rest of this section are ordered executions of the proto$\operatorname{col} \alpha$. Observe that an ordered $r$-round execution $e$ is completely characterized by the inputs to the processors and by specifying the $l$-senders of each processor, for $l=1, \cdots, r$.

The history of a processor in an $r$-round execution $e$ of $\alpha$ is defined by its input value, and the messages it receives in each round $l$ from its $l$-senders, for $l=1, \cdots, r$.

Proposition 1: Let $f$ and $f^{\prime}$ be two $r$-round executions of $\alpha$. Then $P_{k}$ has the same history in $f$ and $f^{\prime}$, if (and only if) in both executions it has the same $r$-senders, $S$, and each processor of $S$ has the same history after the $(r-1)$-st round in $f$ and $f^{\prime}$. (Note that $P_{k}$ belongs to $S$.)

Next we define the solving covering function $C F_{\alpha}$. For a given $\vec{x}$ and $i, C F_{\alpha}\left(\vec{x}^{i}\right)$ is the partial vector $\vec{d}^{i}$ output by the processors $P-\left\{P_{i}\right\}$ in an $s$-round $i$-sleeping execution of $\alpha$ on input $\vec{x}$. (The validity of this definition follows from the fact that $\alpha 1$-solves $T$ in at most $s$ 
rounds, and thus by round $s$ the processors $P-\left\{P_{i}\right\}$ must decide on a covering vector).

We now proceed to the main construction required for our proof, given in Lemma 1 below. This construction uses an idea of [Fe]. First we need the following definition and proposition:

Definition: Two $r$-round executions are adjacent if there are at least $n-1$ processors, each of which has the same history in both executions.

Proposition 2: Let $f$ and $f^{\prime}$ be two $r$-round executions which are identical until round $r-1$, and assume there are two processors, each of which has the same $r$-senders in $f$ and $f^{\prime}$. Then there is a sequence of $n-1 r$-round executions, $\operatorname{CHAIN}\left(f, f^{\prime}\right)=\left(f=f_{1}, f_{2}, \cdots, f_{n-1}=f^{\prime}\right)$ such that $f_{k}$ and $f_{k+1}$ are adjacent for $k=1 . . n-2$.

Proof: For simplicity, assume that the two processors that have the same $r$-senders in $f$ and $f^{\prime}$ are $P_{1}$ and $P_{n}$. Let the $r$-senders of the processors $P_{1}, \cdots, P_{n}$ in execution $f$ be $Q_{1}, Q_{2}, \cdots, Q_{n-1}, Q_{n}\left(Q_{i}\right.$ is the $r$-senders of $\left.P_{i}\right)$, and let the $r$-senders of the processors in execution $f^{\prime}$ be $Q_{1}, Q_{2}^{\prime}, \cdots, Q_{n-1}^{\prime}, Q_{n}$. Then $\operatorname{CHAIN}\left(f, f^{\prime}\right)=\left(f=f_{1}, \cdots, f_{n-1}=f^{\prime}\right)$, where for $i=2, \cdots, n-2$, the first $r-1$ rounds of $f_{i}$ are identical to those of $f$ and $f^{\prime}$, and the $r$ senders of the processors $P_{1}, \cdots, P_{n}$ in $f_{i}$ are $Q_{1}, Q_{2}^{\prime}, \cdots, Q_{i}^{\prime}, Q_{i+1}, \cdots, Q_{n}$.

Lemma 1: Let $1 \leq i<j \leq n$ and let $\vec{x} \in X_{T}$ be an input vector. Then for each $r>0$, there exists a sequence $S_{r}=e_{1}, \cdots, e_{D_{r}}$ of $D_{r}=(n-1)^{r} r$-round executions of $\alpha$, satisfying the following:

(a) $\quad e_{1}$ is an $r$-round $i$-sleeping execution on input $\vec{x}$, and $e_{D_{r}}$ is an $r$-round $j$-sleeping execution on input $\vec{x}$.

(b) The executions $e_{k}$ and $e_{k+1}$ are adjacent, for $k=1, \cdots, D_{r}-1$.

Proof: The proof is by induction on $r$. (The base and the first step of the induction are depicted in Figure 2). For the base, $r=1, e_{1}$ is the 1-round $i$-sleeping execution on input $\vec{x}$ in which the 1 -senders of $P_{i}$ is $P-\left\{P_{j}\right\}$, and $e_{n-1}$ is the 1-round $j$-sleeping execution on input $\vec{x}$ in which the 1 -senders of $P_{j}$ is $P-\left\{P_{i}\right\}$. The sequence $e_{1}, \cdots, e_{n-1}$ is $\operatorname{CHAIN}\left(e_{1}, e_{n-1}\right)$, which satisfies the conditions by Proposition 2 (the assumptions of Proposition 2 hold since $P_{i}$ and $P_{j}$ have each the same 1-senders in $e_{1}$ and $\left.e_{n-1}\right)$.

The induction step: Let $S_{r-1}=e_{1}, \cdots, e_{D_{r-1}}$ be a sequence satisfying the lemma for $r-1$ $\left(D_{r-1}=(n-1)^{r-1}\right)$. Then for each $k=1, \cdots, D_{r-1}-1$ there is a set of $n-1$ processors, which we denote by $Q_{k}$, such that each processor in $Q_{k}$ has the same history in $e_{k}$ and $e_{k+1}$.

We construct the sequence $S_{r}$ by replacing each $(r-1)$-round execution $e_{k}$ in $S_{r-1}$ by a sequence of $n-1 r$-round adjacent executions $e_{k, 1}, e_{k, 2}, \cdots, e_{k, n-1}$; i.e., $S_{r}=e_{1,1}, \cdots, e_{1, n-1}$, $\cdots, e_{D_{r-1}, n-1}$. It remains to define the executions $e_{k, j}$ and to prove that $S_{r}$ indeed satisfies the lemma.

First, to avoid special end-case treatments, we define $Q_{0}=P-\left\{P_{i}\right\}$ and $Q_{D_{r-1}}=P-\left\{P_{j}\right\}$. Now, in $e_{k, 1}$ the first $r-1$ rounds are identical to $e_{k}$. In round $r$ : The $r$-senders of each processor in $Q_{k-1}$ is $Q_{k-1}$, and the $r$-senders of the remaining processor is $Q_{k}$. In $e_{k, n-1}$ the first $r-1$ rounds are also identical to $e_{k}$. In round $r$ : The $r$-senders of each processor in $Q_{k}$ is $Q_{k}$, and the $r$-senders of the remaining processor is $Q_{k-1}$. Now by Proposition 2 we can define the 
sequence $e_{k, 1}, \cdots, e_{k, n-1}$ to be $\operatorname{CHAIN}\left(e_{k, 1}, e_{k, n-1}\right)$.

It remains to show that:

(1) $e_{1,1}$ (i.e., the leftmost execution in $S_{r}$ ) is an $r$-round $i$-sleeping execution and $e_{D_{r-1}, n-1}$ (i.e., the rightmost execution in $S_{r}$ ) is an $r$-round $j$-sleeping execution. This follows immediately by the induction hypothesis and the construction.

(2) Two successive executions in $S_{r}$ are indeed adjacent. If the two executions are in the same CHAIN (that is, they are $e_{k, i}$ and $e_{k, i+1}$ for some $k$ and $i$ ) then this follows from Proposition 2. We now prove that this holds also in the case that these executions are from different CHAINs, i.e. they are of the form $e_{k, n-1}$ and $e_{k+1,1}$ for some $k$. By the construction, until round $r-1 e_{k, n-1}$ is identical to $e_{k}$ and $e_{k+1,1}$ is identical to $e_{k+1}$. By the induction hypothesis, each processor in $Q_{k}$ has the same history in $e_{k}$ and $e_{k+1}$ (and thus has the same history after round $r-1$ in $e_{k, n-1}$ and $\left.e_{k+1,1}\right)$. By the construction, the $r$-senders of each processor in $Q_{k}$, in both $e_{k, n-1}, e_{k+1,1}$ is $Q_{k}$, and thus, by Proposition 1, each of these $n-1$ processors has the same history in $e_{k, n-1}$ and $e_{k+1,1}$.

We now use Lemma 1 to show that $R(T) \leq D_{s}=(n-1)^{s}$. For this, apply Lemma 1 for $r=s$. Then each execution $e_{k}$ defines an output vector $\vec{d}_{k} \in T(\vec{x})$ (each processor decides in $e_{k}$ since it performs round $s$ in $e_{k}$, and $\alpha$ guarantees that a processor that performs round $s$ decides no later than round $s$; the decision value of each processor, and hence the complete output vector, are completely determined since $e_{k}$ is an ordered execution). Statement (a) of the lemma implies that $\vec{d}_{1}$ is an $i$-anchor of $\vec{x}$ which extends $C F_{\alpha}\left(\vec{x}^{i}\right)$, and $\vec{d}_{D_{s}}$ is a $j$-anchor of $\vec{x}$ which extends $C F_{\alpha}\left(\vec{x}^{j}\right)$. Statement (b) of the lemma implies that for every $k, \vec{d}_{k}$ and $\vec{d}_{k+1}$ are either the same vector or are adjacent. Thus, $\left(\vec{d}_{1}, \cdots, \vec{d}_{D_{s}}\right)$ is a path of length at most $D_{s}-1$ from an $i$-anchor to a $j$-anchor of $\vec{x}$. Since this holds for every $i$ and $j, \rho_{C F_{\alpha}}(\vec{x})<D_{s} .{ }^{3}$ Since $\vec{x}$ is arbitrary, we have that $R(T) \leq D_{s}$. This completes the proof of Theorem 1 .

\footnotetext{
${ }^{3} \mathrm{~A}$ tree which contains all anchors with radius at most $D_{s}-1$ can be constructed by a breadth first search starting from any of the anchors.
} 
$\begin{array}{llll}\mathrm{P} 1 & \mathrm{P} 2 & \mathrm{P} 3 & \mathrm{P} 4\end{array}$

$$
\mathrm{r}=1 \longrightarrow \mathrm{r}=2
$$

e11

\begin{tabular}{|l|l|l|l|}
\hline 123 & 123 & 123 & 234 \\
\hline 123 & 123 & 123 & 134 \\
\hline
\end{tabular}

This $\|$ denotes that $\mathrm{P} 2$ has the same history in e11 and e12.

\begin{tabular}{|c|c|c|c|c|}
\hline \multirow{2}{*}{ e12 } & 123 & 123 & 123 & 234 \\
\hline & 134 & 123 & 123 & 134 \\
\hline \multirow{2}{*}{ e13 } & 123 & 123 & 123 & 234 \\
\hline & 134 & 123 & 134 & 134 \\
\hline
\end{tabular}

$\begin{array}{llll}\mathrm{P} 1 & \mathrm{P} 2 & \mathrm{P} 3 & \mathrm{P} 4\end{array}$

e1

\begin{tabular}{|l|l|l|l|}
\hline 123 & 123 & 123 & 234 \\
\hline
\end{tabular}

$\|\quad\| \quad \mathrm{Q} 1=\{1,3,4\}$

e2

\begin{tabular}{|l|l|l|l|}
\hline 123 & 234 & 123 & 234 \\
\hline
\end{tabular}

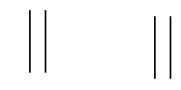

$\| \quad \mathrm{Q} 2=\{1,2,4\}$

e3

\begin{tabular}{|l|l|l|l|}
\hline 123 & 234 & 234 & 234 \\
\hline
\end{tabular}

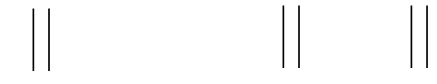

e21 \begin{tabular}{|l|l|l|l|}
\hline 123 & 234 & 123 & 234 \\
\hline 134 & 124 & 134 & 134 \\
\hline
\end{tabular}

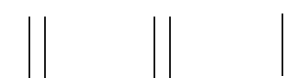

\begin{tabular}{|l|l|l|l|}
\hline 123 & 234 & 123 & 234 \\
\hline 124 & 124 & 134 & 134 \\
\hline
\end{tabular}

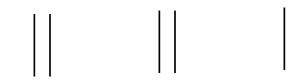

e23

\begin{tabular}{|l|l|l|l|}
\hline 123 & 234 & 123 & 234 \\
\hline 124 & 124 & 134 & 124 \\
\hline
\end{tabular}

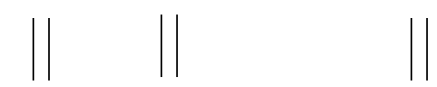

e31

\begin{tabular}{|l|l|l|l|}
\hline 123 & 234 & 234 & 234 \\
\hline 124 & 124 & 234 & 124 \\
\hline
\end{tabular}

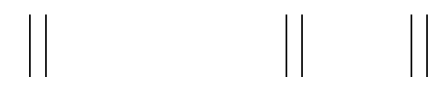

e1 and e11 are 4-sleeping,

e3 and e33 are 1-sleeping.

\begin{tabular}{c|l|l|l|l|}
\hline \multirow{4}{*}{ e32 } & 123 & 234 & 234 & 234 \\
\cline { 2 - 5 } & 124 & 234 & 234 & 124 \\
\hline & $\|$ & $\|$ \\
e33 & 123 & 234 & 234 & 234 \\
\cline { 2 - 5 } & 124 & 234 & 234 & 234 \\
\hline
\end{tabular}

Figure 2: The construction of the sequence for $r=2$ from the sequence for $r-1=1$, for $i=4, j=1$. 


\section{UPPER BOUND}

In this section we present a protocol $\alpha$ that 1 -solves a given bounded task $T$, and has $r c_{\alpha}(T)=2+\left\lceil\log _{(n-1)} R(T)\right\rceil$. The protocol is an improvement of the protocol in [BMZ], whose round complexity is $2+R(T)$ if the number of processes, $n$, is at least 4 , and $2+2 R(T)$ if $n=3$. Like the protocol in [BMZ], this protocol is based on a given solving covering function $C F$. Informally, this protocol differs from the one in [BMZ] in two ways. First, in each execution of this protocol all the vectors that may be suggested by the processors as possible decision vectors belong to a single path in the anchors tree based on $C F$. Second, the convergence to two adjacent vertices on that path is done by an averaging process, similar to the one used in approximate consensus protocols, and not in the step by step fashion of the protocol in [BMZ].

\subsection{The Protocol}

Theorem 2: The round complexity of a bounded task $T$ for $n \geq 3$ processes is at most $2+\left\lceil\log _{(n-1)} R(T)\right\rceil$.

Proof: Let $C F$ be an optimal solving covering function of $T$ (i.e., $R(T)=\underset{\vec{x} \in X_{T}}{\max } \rho_{C F}(\vec{x})$ ). By the computability of $T$, it follows that there is an algorithm TREE that, on input $\vec{x}$, outputs a minimum radius anchors tree $T R E E(x)$ based on $C F$, with a center $\operatorname{ROOT}(\vec{x})$ as its root. Our protocol assumes that each processor has a copy of the algorithms $C F$ and TREE above.

The general outline of the algorithm is as follows: In the first two stages each processor $P_{k}$ is trying to find out the input vector $\vec{x}$. For this, it first broadcasts its input value and receives $n-1$ input values (including its own), which determine a partial input vector $\vec{x}^{j}$ (note that $j \neq k$ ). Then it broadcasts $\vec{x}^{j}$ and waits for $n-1$ such partial vectors. At this point, there are two kinds of processors: those that know only a partial input vector $\vec{x}^{j}$, and hence also know the index $j$ (note that it is the same $j$ for all these processors), and those that know the complete input vector $\vec{x}$.

Now, the processors perform a simple averaging approximate consensus, for $\left\lceil\log _{n-1} R(T)\right\rceil$ rounds, with two kinds of initial values: those that know $\vec{x}^{j}$ start with zero, and those that know $\vec{x}$ start with $R(T)$. During these rounds, each of the processors that knows the complete input vector $\vec{x}$ and/or the index $j$, appends to its messages also these values. After these rounds, each processor will have a value $v$ in $[0, R(T)]$ such that the difference between the maximal and minimal values is at most 1 . If $v$ is equal to zero (in this case $P_{k}$ still knows only $\vec{x}^{j}$ ) then $P_{k}$ decides on $C F\left(\vec{x}^{j}\right)$ (deciding on a (partial) output vector $\left(d_{1}, \cdots, d_{k}, \cdots, d_{n}\right)$ means, in particular, that $d_{k}$ is the decision value of $\left.P_{k}\right)$. Otherwise $P_{k}$ knows $\vec{x}$ (and thus can compute TREE $(\vec{x})$; actually, it will only have to compute ROOT $\vec{x}$ ), or the path in $\operatorname{TREE}(\vec{x})$ from the $j$-anchor to ROOT $(\vec{x}))$. If $v$ is equal to $R(T)$, then $P_{k}$ decides on $\operatorname{ROOT}(\vec{x})$. Otherwise, $P_{k}$ knows $\vec{x}$ and $j$. Then, it "normalizes" the value $v$ to an integer $q$, which is between 0 and the length $l$ of the path from the $j$-anchor to $\operatorname{ROOT}(\vec{x})$. Since $l \leq R(T)$, we have that the difference between the maximal and minimal $q$ values is at most 1 . Finally, each processor decides on the $q$-th vector on this path. Since the difference between the $q$ values is at most 1 , this ensures that each non-faulty processor will decide on one out of two 
adjacent vertices (vectors). This guarantees that the actual output vector is one of these two vectors, and hence it is in $T(\vec{x})$. It is worth mentioning here that deciding on two non-adjacent vectors does not guarantee a legal output vector, and convergence to a single decision vector is actually an agreement, which is impossible by the result of [FLP].

The protocol for $P_{k}$ :

A. BROADCAST $x_{k}$ and WAIT until you RECEIVE $n-1$ stage-A messages

B. you know $\vec{x}^{j}$. BROADCAST $\vec{x}^{j}$ and WAIT until you RECEIVE $n-1$ stage-B messages

C. $\quad$ approximate consensus stage if you know only $\vec{x}^{j}$ then $v \leftarrow 0$ else $v \leftarrow R(T)$

for $r=1$ to $\left\lceil\log _{(n-1)} R(T)\right\rceil$ do

info $\leftarrow \vec{x}$ and / or $j$ (whatever you know of the two)

BROADCAST $(r$, info, $v)$ and WAIT until you RECEIVE $n-1$ messages of round $r$

$v \leftarrow$ the mean of the $n-1 v$ 's received in this round ( ${ }^{*} v$ is a real number*)

end

D. if $v=0$ (you know only $\vec{x}^{j}$ ) then DECIDE $C F\left(\vec{x}^{j}\right)$

else if $v=R(T)$ (you know only $\vec{x}$ ) then DECIDE ROOT $(\vec{x})$

else (you know $\vec{x}$ and $j$ ) do

Let $l$ be the length of the path in TREE $(\vec{x})$ between the $j$-anchor and $\operatorname{ROOT}(\vec{x})$

$q \leftarrow\lfloor v l / R(T)\rfloor$

DECIDE on the $q$ 'th vector of the path in TREE $(\vec{x})$ between the $j$-anchor and ROOT $(\vec{x})$

end

(the $j$-anchor is number 0 in the path, and $\operatorname{ROOT}(\vec{x})$ number $l$ )

HALT

\subsection{Correctness Proof}

It is easy to see that each non-faulty processor eventually decides. We now assume that all processors are non-faulty, and prove that the output vector is legal. By the discussion preceding the protocol, it suffices to prove that for each execution of the protocol in which all processors are non-faulty, there are two adjacent vectors such that each processor decides on one of them. If all the processors know the complete input vector $\vec{x}$ at the end of stage $\mathrm{B}$, then all the processors start and finish stage $\mathrm{C}$ with $v=R(T)$, and decide at stage $\mathrm{D}$ on ROOT $(\vec{x})$, and we are done. Otherwise there exists a unique $j$ such that some processors know only $\vec{x}^{j}$ at the end stage $\mathrm{B}$ (the uniqueness of $j$ is implied by the fact that $n-1$ is a majority).

Denote by $v_{k}$ the value of $v$ that $P_{k}$ holds after $\left\lceil\log _{(n-1)} R(T)\right\rceil$ rounds of approximate consensus in stage $\mathrm{C}$, and by $q_{k}$ the value $q$ it holds after the normalization in stage $\mathrm{D}$. The difference between the maximum and minimum values of the $v_{k}$ 's is at most 1 , since the difference between the $v$ values is initially at most $R(T)$, and it is reduced at least by a factor of $n-1$ each round. ${ }^{4}$

If for all $k, v_{k} \neq R(T)$ and $v_{k} \neq 0$, then each processor $P_{k}$ computes $q_{k}$, and decides on the $q_{k}$-th vector on the path from the $j$-anchor to $\operatorname{ROOT}(\vec{x})$. Clearly the maximum difference between the $q_{k}$ 's is 1 , since $l \leq R(T)$. Hence, all processors decide on two adjacent vectors on

\footnotetext{
${ }^{4}$ This can be shown by considering the collections of $v$ values being averaged in a given round at two different processors: The difference between the sums of $v$ values in each collection is at most the largest difference in the previous round. Thus after dividing by $n-1$, the largest difference is reduced by a factor of at least $n-1$.
} 
that path.

Otherwise, there are two cases where some processor $P_{k}$ decides without computing $q_{k}$ : One case is when $v_{k}=R(T)$ (and $P_{k}$ decides on ROOT $(\vec{x})$ ). In this case all the $v_{i}$ 's are in the range $[R(T)-1, R(T)]$, and the minimum possible $q_{i}$ is $l-1$, which corresponds to a vector adjacent to $\operatorname{ROOT}(\vec{x})$ ). The other case is when $v_{k}=0$, and hence $P_{k}$ decides on $C F\left(\vec{x}^{j}\right)$. In this case, all the $q_{i}$ 's lie in the interval [0,1], and hence all processors decide on $\left.C F \vec{x}^{j}\right)$, or on the $j$-anchor, or on a vector adjacent to the $j$ anchor. This case is equivalent to the case where all processors decide on the $j$-anchor or a vector adjacent to it, since $P_{j}$ never decides on $C F\left(x^{j}\right)$ (it knows its own input $x_{j}$ ), and for every processor other than $P_{j}$, deciding on $C F\left(x^{j}\right)$ is equivalent to deciding on the $j$-anchor.

\section{GENERALIZATION}

In this section we generalize our results for arbitrary tasks. In the general case, the round complexity of a protocol that 1 -solves a (possibly unbounded) task $T$ is not a constant, but a function on the set of input vectors $X_{T}$, as follows.

Definition: Let $T$ be a 1-solvable task. A function $f: X_{T} \rightarrow N$ is a round complexity function of $T$ if there exists a protocol $\alpha$ that 1 -solves $T$, and for each $\vec{x} \in X_{T}, r c_{\alpha}(\vec{x}) \leq f(\vec{x}) \quad\left(r c_{\alpha}(\vec{x})\right.$ is defined in Section 3.2).

Since in general there is no natural total order on such functions, we cannot define the optimal round complexity of a task $T$, but only define the set of minimal round complexity functions of $T$, in the natural partial order of functions, as follows.

Definition: Let $f$ and $g$ be two functions defined on the same domain $X$. Then $f$ is smaller than $g$ if $f \neq g$ and for all $x \in X, f(x) \leq g(x)$. A function $g$ is minimal in a set of function $\boldsymbol{F}$ if there is no $f \in \boldsymbol{F}$ such that $f$ is smaller than $g$.

We define the set of minimal round complexity functions of a task $T$ by a correspondence to the set of minimal radius functions in $\boldsymbol{R}_{T}$ : we show that for each round complexity function $r c$ there exists a radius function $\rho_{C F} \in \boldsymbol{R}_{T}$ such that $\log _{(n-1)} \rho_{C F}$ is smaller than (or equal to) $r c$, and for each radius function $\rho_{C F} \in \boldsymbol{R}_{T}, 3+\left\lceil\log _{(n-1)} \rho_{C F}\right\rceil$ is a round complexity function of $T$.

Thus, the set of functions

$$
\boldsymbol{m} \boldsymbol{R}_{T}=\left\{3+\left\lceil\log _{(n-1)} \rho_{C F}\right\rceil \mid \rho_{C F} \text { is a minimal function in } \boldsymbol{R}_{T}\right\}
$$

approximates the set of minimal round complexity functions of $T$ to within additive constant of 3 , in the following meaning: Each function in $\boldsymbol{m} \boldsymbol{R}_{T}$ is a round complexity function of $T$ such that there is no other round complexity function of $T$ which improves it by more than the additive constant 3 , and for each minimal round complexity function of $T$, there is a function in $\boldsymbol{m} \boldsymbol{R}_{T}$ which is larger by at most 3 .

Theorem 1u: Let $r c$ be a round complexity function of a task $T$. Then, there exists a radius function $\rho_{C F} \in \boldsymbol{R}_{T}$ such that $\log _{(n-1)} \rho_{C F}(\vec{x}) \leq r c(\vec{x})$, for each $\vec{x} \in X_{T}$.

Proof: Since $r c$ is a round complexity function of $T$, there exists a protocol $\alpha$ such that $r c_{\alpha}(\vec{x}) \leq r c(\vec{x})$ for each $\vec{x} \in X_{T}$. From this point the proof is similar to that of Theorem 1 , when 
$s$ is replaced by $r c_{\alpha}(\vec{x})$, and the radius function whose existence is proven is $\rho_{C F}$.

Theorem $2 \mathbf{u}$ : Let $\rho_{C F}$ be a radius function of a task $T$. Then, $3+\left\lceil\log _{(n-1)} \rho_{C F}\right\rceil$ is a round complexity function of $T$.

Proof: We only need few minor changes in the protocol of Section 6: First, all occurrences of $R(T)$ are replaced by $\rho_{C F}(\vec{x})$. Now, the problem is that processors that at the beginning of stage $C$ know only $\vec{x}^{j}$, cannot compute $\left\lceil\log _{(n-1)} \rho_{C F}(\vec{x})\right\rceil$ - the number of approximate consensus rounds. To solve this problem, we add an initialization round in stage $C$ (this idea is borrowed from [DLPSW]) in which a processor that receives a message with $v=0$ sets its own $v$ to 0 , and a processor such that all the $n-1 v$ values it receives are 0 , and hence it still knows only $\vec{x}^{j}$, broadcasts a "FINISH" message and exits stage C. A processor that receives in the next rounds a "FINISH" message, sets its $v$ to 0 , broadcasts a "FINISH" message and exits stage C. Thus, if some processor broadcasts "FINISH" message in the initialization round, then all processors set their $v$ to 0 , and it follows that all the $v$ 's will be zero after stage $\mathrm{C}$. The rest of the correctness proof is similar to the one in Section 6.

\section{APPLICATIONS}

We present here new optimal bounds on the round complexity of the 1-solvable tasks mentioned in the paper. The first three examples deal with bounded tasks, and provide upper bounds of 3 rounds for the tasks involved (it can be shown that 2 rounds are not enough). All previous protocols that 1 -solved these tasks required $\Omega(n)$ rounds. The bounds are proved by presenting a covering function $C F$ for each task $T$ which prove that $R(T) \leq n-1$ (and hence $\log _{n-1} R(T) \leq 1$ ). Actually, each of the covering functions presented will be optimal. The last example deals with the strong binary monotone approximate consensus, and provide a bound of $4+\log _{n-1}\left(\frac{d-c}{\varepsilon}\right)$, where $d$ and $c$ are the two medians of the numbers of the input vector. This is approximately the same bound that is proved optimal in [Fe] for the task of approximate consensus, which seems to be considerably simpler than the strong binary monotone approximate consensus. (We note, however, that the bounds in [Fe] apply to multiple failures.)

The formal definitions of the tasks discussed below are given in Section 2.2.

(1) Strong Binary Monotone Consensus: Let $\vec{x}^{i}=\left(x_{1}, \cdots, x_{i-1}, *, x_{i+1}, \cdots, x_{n}\right)$ be a partial input vector for this task. Again, we assume for simplicity that $n$ is even. In this case there is a unique possible covering function $C F$, defined by $C F\left(\vec{x}^{i}\right)=(c, \cdots, c)$, where $c$ is the median of the multiset $\left\{x_{1}, \cdots, x_{i-1}, x_{i+1}, \cdots, x_{n}\right\}$.

We now describe anchors trees based on $C F$. For a given input vector $\vec{x}$, let $c$ and $d$ be the two medians of the multiset $\left\{x_{1}, \cdots, x_{n}\right\}$. If $c=d$ then the anchors tree consists of the single vertex $(c, \cdots, c)$. Otherwise, it consists of the path $[(c, \cdots, c, d),(c, \cdots, c, d, d)$ $\cdots,(c, d, \cdots, d)]$. In the first case the radius of the tree is 0 , and in the second is $\frac{n}{2}-1$. It can be shown that this anchor tree is of minimum possible radius, and hence $R(T)=\frac{n}{2}-1$. 
(2) Renaming with $n+1$ new names: In this task the input to each processor is its id, and the id's are not mutually known. Such a task cannot be modeled as a function from input vectors to output vectors, since there is no fixed order among the processes. Instead, it is modeled as a function between input sets to allowed output sets [BMZ]. By adapting the definitions for this model, as is done in [BMZ], we get that $R(T) \leq n-1$.

(3) Order Preserving Renaming with $2 n-1$ new names: This task is order invariant, i.e.: $T(\vec{x})$ depends only on the relative order among the entries of $\vec{x}$. $C F$ is also order invariant, and we describe $C F\left(\vec{x}^{i}\right)$ only for the case that the entries in $\vec{x}$ are monotone increasing (i.e., $\left.x_{i}<x_{i+1}\right)$. The adaptation of the definition to other order types is straightforward. In this case, $C F\left(\vec{x}^{i}\right)=(2,4, \cdots, 2 i-2, *, 2 i, \ldots, 2 n-2)$. A suitable anchors tree of such $\vec{x}$ is the path of length $2 n-2$ (and hence of radius $n-1$ ) starting at the 1 -anchor $(1,2,4, \cdots, 2 n-2)$ and ending at the $n$-anchor $(2,4, \cdots, 2 n-2,2 n-1)$, that passes via all the $i$-anchors. (E.g., for $n=3$ this path is $[(1,2,4),(1,3,4),(2,3,4),(2,3,5),(2,4,5)]$.)

(4) Strong Binary Monotone Approximate Consensus (for a given $\varepsilon$ ): The input, and the (unique) covering function $C F$ is the same as for the binary monotone consensus. The minimal radius anchors tree based on $C F$ is also similar to the one for the binary consensus, but this time $\varepsilon$ must be taken into account:

For a given input vector $\vec{x}$, let $c$ and $d$ be the two medians of the multiset $\left\{x_{1}, \cdots, x_{n}\right\}$. Assume for simplicity that $\varepsilon$ divides $d-c$. If $c=d$ then the anchors tree consists of the single vertex $(c, \cdots, c)$. Otherwise, it consists of the path $[(c, \cdots, c, c+\varepsilon)$, $(c, \cdots, c, c+\varepsilon, c+\varepsilon), \cdots,(c, c+\varepsilon, \cdots, c+\varepsilon),(c+\varepsilon, \cdots, c+\varepsilon), \cdots,(d-\varepsilon, \cdots, d-\varepsilon, d-\varepsilon)$, $\cdots,(d-\varepsilon, d, \cdots, d)]$. In the first case the radius of the tree is 0 , and in the second is

$n\left(\frac{d-c}{\varepsilon}\right)$. Thus, the upper bound provided by our results (for unbounded tasks) is at most $5+\log _{n-1}\left(\frac{d-c}{\varepsilon}\right)$.

Acknowledgements: We would like to thank two anonymous referees for their helpful comments.

\section{REFERENCES}

[ABDKPR] H. Attiya, A. Bar-Noy, D. Dolev, D. Koller, D. Peleg, R. Reischuk, Achievable cases in an asynchronous environment, Proc. of the 28th FOCS, 1987, pp. 337-346. A new version appears in Journal of the ACM, Vol. 37 no. 3, 1990, pp. 524-548.

[ADG] H. Attiya, D. Dolev and J. Gil, Asynchronous Byzantine consensus, Proc. of the 3rd PODC, 1984, pp. 119-133.

[ALS] H. Attiya, N. Lynch and N. Shavit, Are wait-free algorithms fast ?, Proc. of the 31th FOCS, 1990, pp. 422-427.

[BG] E. Borowski and E. Gafni. Generalized FLP impossibility result for t-resilient asynchronous computations, Proc. 25th ACM STOC, 1993.

[BMZ] O. Biran, S. Moran and S. Zaks, A combinatorial characterization of the distributed tasks which are solvable in the presence of one faulty processor, Journal of Algorithms 11, 1990, pp. 420-440. 
[DLS] C. Dwork, N. Lynch and L. Stockmeyer, Consensus in the presence of partial synchrony, Journal of the ACM, Vol. 35 no. 2, 1988, pp. 288-323.

[DLPSW] D. Dolev, N. A. Lynch, S. Pinter, E. Stark and W. Weihl, Reaching approximate agreement in the presence of faults, Journal of the ACM, Vol. 33 no. 3, 1986, pp. 499-516.

[Fe] A. D. Fekete, Asynchronous Approximate Agreement, Proc. of the 6th PODC, 1987, pp. 64-76.

[FLP] M. J. Fischer, N. A. Lynch and M. S. Paterson, Impossibility of distributed consensus with one faulty process, Journal of the ACM, Vol. 32 No. 2 (1985), pp. 373-382.

[HS] M. Herlihy and N. Shavit The asynchronous computability theorem for t-resilient tasks, Proc. 25th ACM Symp. on Theory of Computing, 1993.

[HT] M. Herlihy and M. Tuttle, Wait free computation in message-passing systems, Proc. of the 9th PODC, 1990, pp. 347-362.

[KMZ] E. Korach, S. Moran and S. Zaks, Tight lower and upper bounds for some distributed algorithms for a complete network of processors, Proc. of the 3rd PODC, 1984, pp. 199-207.

[MW] S. Moran and Y. Wolfstahl, Extended impossibility results for asynchronous complete networks, Information Processing Letters, 26, 1987, pp. 145-151.

[NT] G. Neiger and S. Toueg, Automatically increasing the fault-tolerance of distributed systems, Proc. of the 7th PODC, 1988, pp. 248-262.

[TKM] G. Taubenfeld, S. Katz and S. Moran, Initial failures in distributed computations, Journal of Parallel Programming 18:4, 1989, pp. 255-273.

[SZ] M. Saks and F. Zaharoglou, Wait-free k-set agreement is impossible: The topology of public knowledge, Proc. 25th ACM Symp. on Theory of Computing, 1993. 